\title{
Neural Correlates of Impaired Reward-Effort Integration in Remitted Bulimia Nervosa
}

\author{
Stefanie Verena Mueller', Yosuke Morishima ${ }^{2}$, Simon Schwab ${ }^{3,4}$, Roland Wiest ${ }^{5}$, Andrea Federspiel ${ }^{4}$ and \\ Gregor Hasler*,I
}

'Division of Molecular Psychiatry, Translational Research Center, University Hospital of Psychiatry, University of Bern, Bern, Switzerland; ${ }^{2}$ Division of Systems Neuroscience of Psychopathology, Translational Research Center, University Hospital of Psychiatry, University of Bern, Bern, Switzerland; ${ }^{3}$ Big Data Institute, Li Ka Shing Centre for Health Information and Discovery, Nuffield Department of Population Health, University of Oxford, Oxford, UK; ${ }^{4}$ Psychiatric Neuroimaging Unit, Translational Research Center, University Hospital of Psychiatry, University of Bern, Bern, Switzerland; ${ }^{5}$ Support Center for Advanced Neuroimaging (SCAN), Institute for Diagnostic and Interventional Neuroradiology, University Hospital Inselspital and University of Bern, Bern, Switzerland

\begin{abstract}
The integration of reward magnitudes and effort costs is required for an effective behavioral guidance. This reward-effort integration was reported to be dependent on dopaminergic neurotransmission. As bulimia nervosa has been associated with a dysregulated dopamine system and catecholamine depletion led to reward-processing deficits in remitted bulimia nervosa, the purpose of this study was to identify the role of catecholamine dysfunction and its relation to behavioral and neural reward-effort integration in bulimia nervosa. To investigate the interaction between catecholamine functioning and behavioral, and neural responses directly, 17 remitted bulimic ( $\mathrm{rBN}$ ) and 21 healthy individuals (HC) received alpha-methyl-paratyrosine (AMPT) over $24 \mathrm{~h}$ to achieve catecholamine depletion in a randomized, crossover study design. We used functional magnetic resonance imaging ( $\mathrm{PMRI}$ ) and the monetary incentive delay (MID) task to assess reward-effort integration in relation to catecholaminergic neurotransmission at the behavioral and neural level. AMPT reduced the ability to integrate rewards and efforts effectively in $\mathrm{HC}$ participants. In contrast, in rBN participants, the reduced reward-effort integration was associated with illness duration in the sham condition and unrelated to catecholamine depletion. Regarding neural activation, AMPT decreased the reward anticipation-related neural activation in the anteroventral striatum. This decrease was associated with the AMPT-induced reduction of monetary earning in $\mathrm{HC}$ in contrast to rBN participants. Our findings contributed to the theory of a desensitized dopaminergic system in bulimia nervosa. A disrupted processing of reward magnitudes and effort costs might increase the probability of maintenance of bulimic symptoms. Neuropsychopharmacology (2018) 43, 868-876; doi:10.1038/npp.2017.277; published online 20 December 2017
\end{abstract}

\section{INTRODUCTION}

Binge eating episodes followed by inappropriate compensatory behavior are the cardinal symptoms of bulimia nervosa (BN). As food consumption is considered a rewarding behavior, previous studies often used food stimuli to assess reward processing in BN (Frank, 2013; Wierenga et al, 2014). These studies revealed that participants with $\mathrm{BN}$ showed a reduced activation in reward-related brain regions during anticipation of food rewards (Bohon and Stice, 2011; Frank et al, 2011). The dopaminergic system is importantly related to reward processing (Schultz, 2016). Accordingly, Frank suggested that the dopaminergic system play a core role in the pathophysiology of BN (Frank, 2016). Frank proposed

*Correspondence: Professor G Hasler, Devision of Molecular Psychiatry, Translational Research Center, University Hospital of Psychiatry, University of Bern, University Hospital of Psychiatry, Bolligenstrasse I I I, Bern 60, 3000 Switzerland, Tel: +4I 31 93099 74, Fax: +4I 31 93099 61, E-mail: gregor.hasler@puk.unibe.ch

Received 13 July 2017; revised 26 September 2017; accepted 30 October 2017; accepted article preview online 6 November 2017 that $\mathrm{BN}$ is related to a desensitized dopaminergic system (Frank, 2013, 2016): Individuals with BN responded with a reduced activation of the ventral striatum and insula to an unexpected delivery and omission of a sucrose solution (Frank et al, 2011). To address the functional role of the catecholaminergic system in reward processing in $\mathrm{BN}$, we investigated the impact of experimental catecholamine depletion on reward learning in remitted bulimia nervosa $(\mathrm{rBN})$ in a previous study (Grob et al, 2012). Following catecholamine depletion, rBN participants showed rewardlearning deficits contrary to healthy control (HC) participants (Grob et al, 2012). The findings of this study provided evidence for a causative relationship between catecholaminergic neurotransmission and reward-processing deficits in BN. Nonetheless, the functional role of catecholamine dysfunctions and their impact on reward processing for guiding behavior in $\mathrm{BN}$ has not yet been determined.

Although monetary rewards are secondary reinforcers (Hasler, 2012; Knutson and Heinz, 2015), studies have demonstrated that they are qualified to probe the reward system in BN (Grob et al, 2012). The 'monetary incentive delay' (MID) 
task was developed to investigate reward anticipation and reward-effort relationships (Knutson et al, 2001a, 2001b). In detail, a successful performance of this task requires an effective integration of reward magnitudes and effort costs during task execution. An appropriate indicator of an effective reward-effort integration is the monetary earning in this task: To maximize monetary gains, the reaction time needs to be decreased (ie, to invest more effort) after anticipating large monetary gains or losses. An earlier study revealed that the monetary earning was reduced in individuals with major depressive disorder (MDD) during a current depressive episode relative to healthy individuals, whereas remitted MDD and healthy individuals showed no difference (DelDonno et al, 2015).

Dopamine neurons of the primate ventral midbrain have been reported to reflect the probability and magnitude of rewards in a probabilistic reward task: The phasic activity of dopamine neurons increased after a reward predictive stimuli in relation to the reward probability (Fiorillo et al, 2003). Therefore, this activation of dopaminergic neurons during the anticipation of rewards plays an important role in a successful task performance (Hasler, 2012). In addition, dopamine has been reported to be related to effortful behavior: In animals, dopamine depletion in the nucleus accumbens resulted in a reduced performance of high-effort behavior (Niv et al, 2007; Salamone et al, 2007). Functional imaging studies using the MID task consistently revealed that the activation of the anteroventral striatum (AVS) showed an increased activation in response to large relative to small monetary gains (Knutson et al, 2001a). Regarding eating disorders, obese participants experiencing binge eating episodes showed a reduced neural response to monetary gain in the ventral striatum (Balodis et al, 2013).

With this study, we addressed the direct relationship between catecholaminergic neurotransmission and the ability to integrate reward and effort effectively for behavioral guidance in $\mathrm{BN}$. In a pharmacological challenge study, $\mathrm{rBN}$ and $\mathrm{HC}$ participants performed the MID task during functional magnetic resonance imaging (fMRI) after catecholamine or sham depletion had been induced. Following Frank's hypothesis of a desensitized dopaminergic system in BN (Frank, 2016) and our previous study (Grob et al, 2012), we hypthesized that catecholamine depletion will have a lesser effect on reward-effort integration in $\mathrm{rBN}$ than in HC participants. This effect is first indicated by changes of the monetary earning in the MID task, and second by neural activation changes during anticipation of monetary gains or losses in the AVS, the well-defined core region in this task (Knutson et al, 2001a). We hypothesized that catecholamine depletion would dampen the reward anticipation-related activation of the AVS, and that this reduction of the anticipation signal would be less pronounced in $\mathrm{rBN}$ compared to $\mathrm{HC}$ participants.

\section{MATERIALS AND METHODS}

\section{Participants}

Seventeen female participants in remission from $\mathrm{BN}(\mathrm{rBN})$, and 21 female healthy volunteers (HC) were included in this

Table I Characteristics

\begin{tabular}{|c|c|c|c|c|}
\hline Characteristics/clinical ratings & HC participants & rBN participants & T-statistic & p-value \\
\hline Age, mean $\pm S D$, years & $27.3 \pm 9.4$, range $20-53$ & $29.6 \pm 8.9$, range $20-49$ & $t_{35.0}=-0.76$ & $p=0.46$ \\
\hline Years of education, mean $\pm \mathrm{SD}$, years & $|5| \pm 2.4$. & $15.2 \pm 2.8$ & $t_{31.5}=-0.12$ & $p=0.91$ \\
\hline Body mass index $(\mathrm{BMI})$, mean $\pm \mathrm{SD}, \mathrm{kg} / \mathrm{m}^{2}$ & $\begin{array}{l}24.2 \pm 3.3 \text {, range } \\
19.3-32.2\end{array}$ & $\begin{array}{c}21.6 \pm 2.3, \text { range } \\
18.4-27.6\end{array}$ & $t_{35.1}=2.85$ & $p=0.008$ \\
\hline Age at onset of $\mathrm{BN}$, mean $\pm \mathrm{SD}$, years & NA & $18.9 \pm 5.5$, range $12-35$ & & \\
\hline Time in remission from $\mathrm{BN}$, mean $\pm \mathrm{SD}$, months & NA & $\begin{array}{l}47.1 \pm 46.5, \text { range } \\
4-146\end{array}$ & & \\
\hline Major depression during and/or after $\mathrm{BN}$, (during, after), $\mathrm{n}$ & NA & $12(11,3)$ & & \\
\hline Time in remission from $M D D$, mean $\pm S D$, months & & $\begin{array}{l}55.4 \pm 63.1, \text { range } \\
6-228\end{array}$ & & \\
\hline Time medication free, mean $\pm S D$, months & & $\begin{array}{l}52.0 \pm 45.1, \text { range } \\
10-122\end{array}$ & & \\
\hline $\begin{array}{l}\text { EDE-Q global score }- \text { past ( } 4 \text { weeks during acute phase with most severe bulimic } \\
\text { symptoms), mean } \pm S D \text {, scores }\end{array}$ & NA & $4.38 \pm 0.9$ & & \\
\hline EDE-Q global score - screening ( 4 weeks before screening), mean $\pm S D$, scores & $0.57 \pm 0.5$ & $1.37 \pm 1.01$ & $t_{22.3}=-2.97$ & $p=0.008$ \\
\hline MADRS, mean $\pm S D$, scores & $1.19 \pm 1.99$ & $3.24 \pm 3.85$ & $t_{22.8}=-1.99$ & $p=0.06$ \\
\hline
\end{tabular}

Abbreviations: AN, anorexia nervosa; BMl, body mass index; BN, bulimia nervosa; EDE-Q, Eating Disorder Examination-Questionnaire (Hilbert and Tuschen-Caffier, 2006); HC, healthy control participants; MADRS, Montgomery-Åsberg Depression Rating Scale (Schmidtke et al, 1988); MDD, major depressive disorder; n, number;

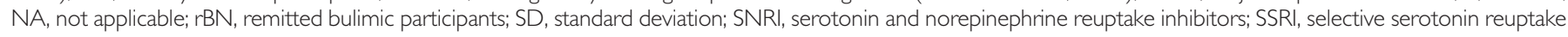
inhibitors; TCA, tricyclic antidepressants.

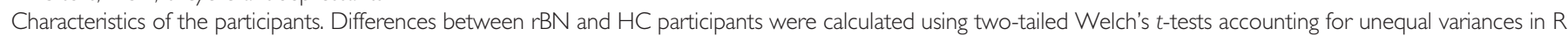
(version 3.3.3) (R Foundation for Statistical Computing, 2017). 
study. All rBN participants had met the DSM-IV criteria of $\mathrm{BN}$ in past bulimic episodes and had been in remission without any binge eating or purging episode for at least four months. The inclusion and exclusion criteria for both groups, and the recruitment procedure were described previously (Mueller et al, 2017). None of our participants showed binge or restrictive eating behavior. The protocol of this study and the written informed consent were approved by the local ethics committee of Canton Bern, Switzerland, and performed in accordance with the principles of the Declaration of Helsinki.

\section{Procedure}

The procedure of this study has been reported previously (Mueller et al, 2017). In short, all participants were first invited to a screening visit including the Structural Clinical Interview for DSM-IV (First et al, 2002), a diagnostic interview with a psychiatrist, and a physical examination at the University Hospital of Psychiatry in Bern. Clinical and demographical characteristics of both groups are presented in Table 1. Second, all participants underwent two identical experimental sessions, which took place at the Inselspital, University of Bern, and included magnetic resonance (MR) imaging and blood sampling. In a double-blind, crossover study design, the participants received once catecholamine depletion induced by alpha-methyl-paratyrosine (AMPT) and once sham depletion in a randomized order. AMPT was provided to the participants in a body weight adjusted dose ( $40 \mathrm{mg} / \mathrm{kg}$ body weight, to a maximum of $4 \mathrm{~g}$ ), and was administered at four time points over $24 \mathrm{~h}$ (Figure 1), to avoid any adverse reactions. In the sham condition, the participants received $25 \mathrm{mg}$ diphenhydramine at the first and placebo at the remaining three time points. Diphenhydramine was administered in the sham condition to increase blinding between the two sessions. Previous studies reported that diphenhydramine is very efficient to use for the sham condition, as it causes similar sedation, but no symptoms in contrast to AMPT (Bremner et al, 2003; Lam et al, 2001; Neumeister et al, 1998). The two experimental sessions were separated by at least 7 days to avoid any crossover effects. Serum prolactin levels were measured in blood samples as a proxy of the depth of catecholamine depletion.
Follow-up telephone interviews were conducted with a latency varying between 18 and 42 months after study participation. In these interviews, bulimic relapses defined by at least one binge eating or purging episode were assessed.

\section{MID Task}

During $\mathrm{AMRI}$, the participants performed a modified version of the well-established MID task described by Knutson et al, 2001a, 2001b. The software E-prime (Psychology Software Tools, Pittsburgh, PA, 2014) was used to run the MID task (Figure 1). In this task, each trial started with the presentation of one of five different cues that indicated potential gain or loss of different amounts of money ( 0.20 or 2.00 Swiss francs) or no outcome. After a variable interval (delay period), the participants had to respond with a key press to a target square appearing for variable duration. Afterwards, the feedback informed the participants on their monetary gain or loss in the current trial and on their cumulative total earning at that point. The trials were presented in a counterbalanced order with a variable interstimulus interval optimized by optseq2 software (https:// surfer.nmr.mgh.harvard.edu/optseq). A detailed description of this task is provided in the Supplementary Information. After completing both experimental sessions, one of the two sessions was randomly selected and the participants received the monetary earning in that session additionally to 300 Swiss francs for the participation in the whole experiment. Selecting randomly only one session ensured a similar motivation to perform this task effectively in both sessions to increase the total monetary earning. Consequently, the monetary earning in this task indicates the effectiveness of integrating reward magnitudes and effort costs to guide behavioral performance.

\section{Analysis of the Behavioral Data and Serum Prolactin Level}

The behavioral data and serum prolactin level were analyzed with mixed effect model analyses using the 'lmer' method of the 'Ime4' package (Bates et al, 2015) and the 'ImerTest' package (Kuznetsova et al, 2016), providing $p$-values in $\mathrm{R}$ (version 3.3.3) ( $\mathrm{R}$ Foundation for Statistical Computing,

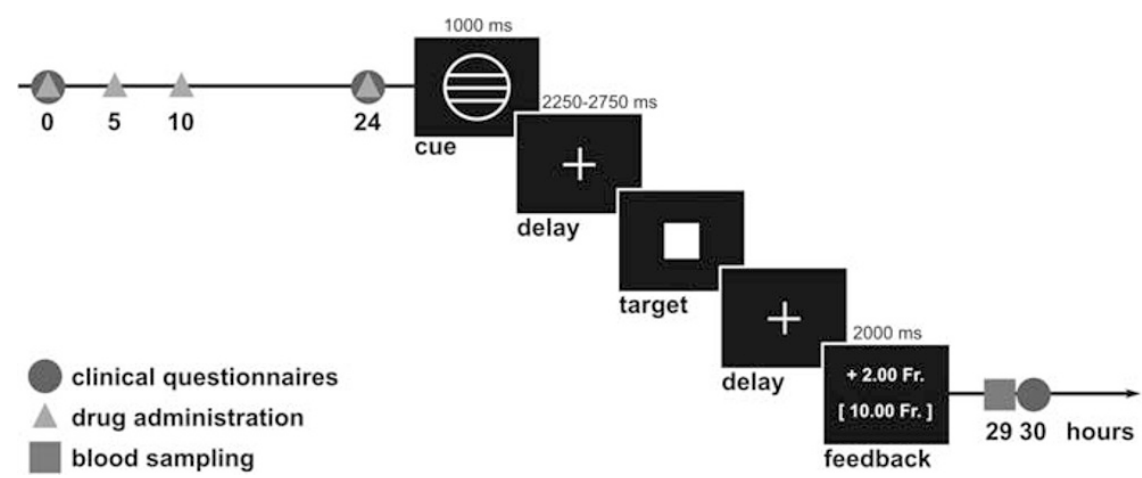

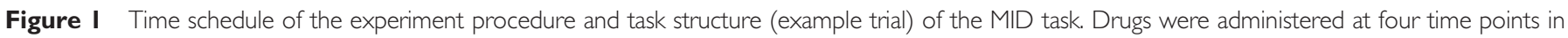

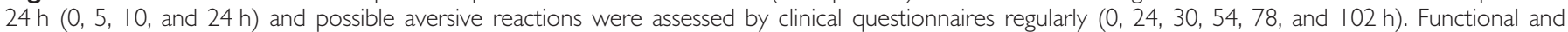

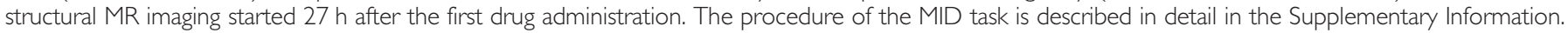
Blood samples were collected after MR imaging ( $29 \mathrm{~h}$ after first drug administration). 
2017). Correlation analysis were conducted with the 'rcorr' method of the 'Hmisc' package (Harrell, 2016). The statistical significance level was set at $\alpha=0.05$.

The models for analyzing the influence of AMPT on the monetary earning, the mean reaction time in successful trials, the proportion of successful key presses (hit rate), and on serum prolactin levels included group ( $\mathrm{rBN}$ vs $\mathrm{HC}$ participants) and drug (AMPT vs sham depletion) as fixed effects. A random effect term modeled a random intercept for each participant. In addition, to assess the effect of the different valences (anticipation of monetary gain $v s$ loss) and magnitudes (anticipation of 2 vs 0.20 Swiss francs) on the reaction time and hit rate, we conducted further mixed effect model analyses including group, drug, valence, and magnitude as fixed effects. A detailed description of these analyses is provided in the Supplementary Information.

To identify possible associations between clinical characteristics and behavioral performance in rBN participants, we conducted correlation analyses between the monetary earning in the sham condition, and illness and remission duration (two-tailed, $p<0.05$ ). Because the illness and remission duration were not normally distributed, we applied a Spearman's rho rank correlation. The illness duration was defined as the total duration of active illness characterized by the diagnostic criteria of $\mathrm{BN}$. The duration between the last binge eating or purging behavior and the participation in the study described the remission duration.

\section{MR Imaging}

In each session, functional and anatomical MR images were acquired on a 3 Tesla Siemens Magnetom Trio Scanner (Erlangen, Germany) with a 12-channel regular head coil. The parameters used for acquiring the functional and anatomical MR images are provided in the Supplementary Information.

\section{fMRI Analysis}

The fMRI data were analyzed using Statistical Parametric Mapping (SPM12, Wellcome Trust Center for Neuroimaging, University College London, http://www.fil.ion.ucl.ac. $\mathrm{uk} / \mathrm{spm} /$ ). The preprocessing and first-level analysis using the General Linear Model of the imaging data were described in the Supplementary Information.

To identify the effect of catecholamine depletion on neural activation during the anticipation phase, we included the contrast images 'large $v s$ small monetary gain anticipation,' and 'large $v s$ small monetary loss anticipation' in separate second-level analyses. These flexible factorial analyses included group as between-subject, drug as within-subject factor, and a random factor for each participant. For our region of interest (ROI), we applied small volume correction using an explicit mask of the bilateral anteroventral striatum (AVS). The definition of the AVS is based on the previous report of Drevets et al, 2001 and is described in the Supplementary Information. The statistical significance threshold of $p<0.05$, family-wise error (FWE)-small volume corrected, was determined for these analyses.

To assess the relation between the AMPT effect on neural activation in the AVS and monetary earning during the MID task, we calculated Pearson's correlation coefficients between the AMPT-induced changes in the monetary earning and the AMPT-induced reward anticipation-related activation changes in the AVS (two-tailed, $p<0.05$ ) separately for each participant group. The AMPT effect on neural activation in this region was calculated in the following way: In the flexible factorial design described above, we identified a peak voxel in the AVS revealing a main effect of the drug conditions in both participant groups (Montreal Neurological Institute (MNI)-coordinate $\left.\left[\begin{array}{lll}x & y & z\end{array}\right]=\left[\begin{array}{lll}-12 & 12 & -8\end{array}\right]\right)$. From a sphere (radius $=4 \mathrm{~mm}$ ) centered on this peak voxel, we first extracted the mean beta estimates of the contrast between anticipation of large and small monetary gain to obtain the mean reward anticipation-related neural activation in each drug condition. Second, we subtracted the mean reward anticipation-dependent neural activation of the sham condition from that following AMPT. In a similar manner, the AMPT-induced behavioral changes were acquired by the subtraction of the monetary earning during sham condition from the earning following AMPT.

Additionally, we conducted correlation analyses between the monetary earning or the mean beta estimates of the $4 \mathrm{~mm}$-sphere in the left AVS and body mass index (BMI), age, depressive and eating disorder symptoms at the screening visit and during the most severe bulimic period in past active illness of the rBN participants. Further, to examine any influence of the arousal or alertness of the participants on the behavioral performance and neural activation, we calculated correlation analyses between AMPT-induced alterations on the vigor and fatigue subscales of the Profile of Mood States (POMS) (McNair et al, 1992) and AMPT-induced changes of monetary earning or reward anticipation-related neural activation in the left AVS. Detailed descriptions of these correlation analyses are provided in the Supplementary Information.

\section{RESULTS}

\section{Behavioral Performance}

The analysis of the monetary earning revealed no significant main effect of the drug conditions $\left(\mathrm{F}_{1,36}=1.21 ; p=0.28\right)$ and of the two groups $\left(\mathrm{F}_{1,36}=0.95 ; p=0.34\right)$. Nonetheless, a significant group-by-drug interaction was found $\left(\mathrm{F}_{1,36}=4.71\right.$; $p=0.037$; Figure 2a): HC participants earned less money following AMPT relative to the sham condition, whereas the monetary earning of rBN participants was not influenced by AMPT (Table 2). In addition, HC participants earned significantly more money than $\mathrm{rBN}$ participants in the sham condition, but not following AMPT.

The monetary earning in the sham condition was found to be significantly correlated with the illness duration in $\mathrm{rBN}$ participants: the longer the duration of active illness, the less money they earned in the sham condition (rho $=-0.51$, $p=0.039$; Figure $2 \mathrm{~b}$ ). In contrast, remission duration was not significantly associated with monetary earning in the sham condition ( $\mathrm{rho}=-0.15, p=0.56$ ).

Both groups showed an increased reaction time $\left(\mathrm{F}_{1,36}=18.55, p=0.0002\right)$ in successful trials and a reduced hit rate $\left(\mathrm{F}_{1,36}=19.05, p=0.0002\right)$ following AMPT. The two groups did not differ in these behavioral parameters (reaction time: $\mathrm{F}_{1,36}=0.30, p=0.59$; hit rate: $\mathrm{F}_{1,36}=1.71$, $p=0.20$ ) and we did not find a significant group-by-drug 

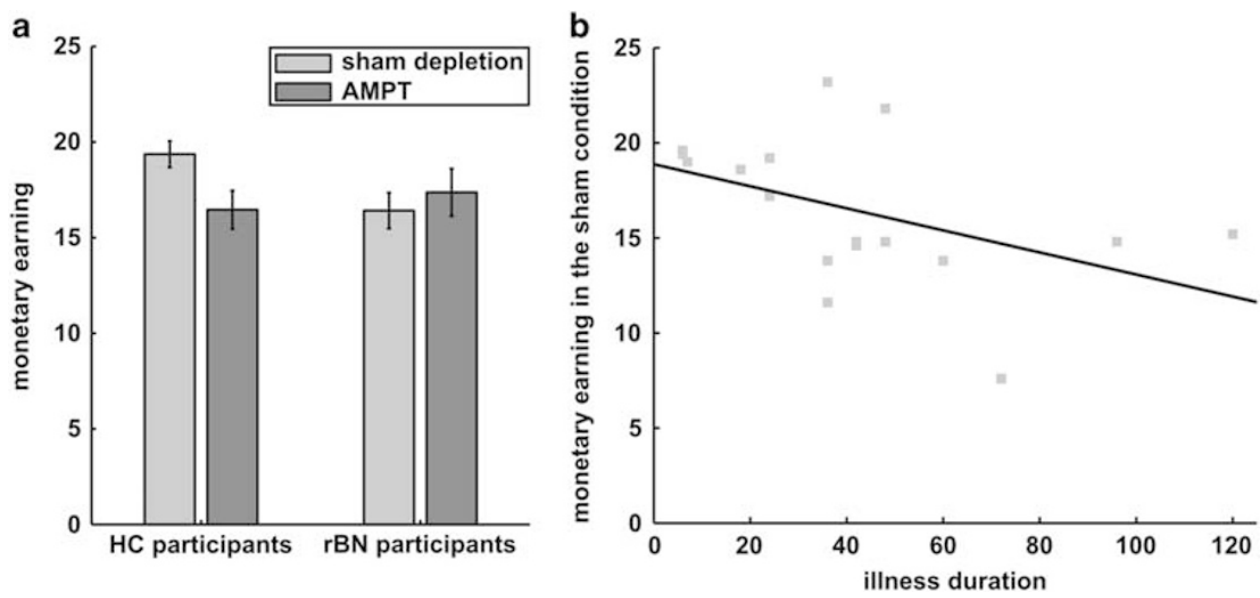

Figure 2 Monetary earning. (a) Mean (bars) and standard error (error bars) of the monetary earning in both participant groups (rBN and HC participants) and drug conditions (catecholamine and sham depletion). Catecholamine depletion was induced by the administration of AMPT. (b) Scatter plot of the amount of money earned in the sham condition and illness duration in rBN participants (Spearman's rho rank correlation: rho $=-0.5 \mathrm{I}, p=0.039$ ).

Table 2 Behavioral Performance and Serum Prolactin Levels

\begin{tabular}{|c|c|c|c|c|c|c|c|c|c|}
\hline \multirow[b]{2}{*}{$\begin{array}{l}\text { Behavioral } \\
\text { performance }\end{array}$} & \multirow[b]{2}{*}{$\begin{array}{l}\text { Drug } \\
\text { condition }\end{array}$} & \multirow[b]{2}{*}{$\begin{array}{c}\text { HC } \\
\text { participants }\end{array}$} & \multirow[b]{2}{*}{$\begin{array}{c}\text { rBN } \\
\text { participants }\end{array}$} & \multicolumn{2}{|c|}{ Two-sample $t$-test } & \multicolumn{2}{|c|}{$\begin{array}{l}\text { HC-participants: } \\
\text { paired t-test }\end{array}$} & \multicolumn{2}{|c|}{$\begin{array}{l}\text { rBN-participants: } \\
\text { paired } t \text {-test }\end{array}$} \\
\hline & & & & T-statistic & $P$-value & $T$-statistic & $P$-value & T-statistic & $P$-value \\
\hline Monetary earning, & Sham depletion & $19.36 \pm 3.2$ & $|6.4| \pm 3.9$ & $t_{30.8}=2.52$ & $p=0.017$ & $t_{20}=2.57$ & $p=0.019$ & $t_{16}=-0.68$ & $p=0.51$ \\
\hline Swiss francs & AMPT & $16.46 \pm 4.6$ & $17.36 \pm 5.1$ & $t_{32.5}=-0.57$ & $p=0.58$ & & & & \\
\hline Hit rate, \% & $\begin{array}{l}\text { Sham depletion } \\
\text { AMPT }\end{array}$ & $\begin{array}{l}65.2 \pm 2.2 \\
63.4 \pm 2.7\end{array}$ & $\begin{array}{l}64.4 \pm 1.5 \\
62.5 \pm 2.9\end{array}$ & $\begin{array}{l}t_{34.6}=1.39 \\
t_{33.5}=0.98\end{array}$ & $\begin{array}{l}p=0.18 \\
p=0.34\end{array}$ & $t_{20}=3.8 \mathrm{I}$ & $p=0.002$ & $t_{16}=2.56$ & $p=0.022$ \\
\hline Serum prolactin level & $\begin{array}{l}\text { Sham depletion } \\
\text { AMPT }\end{array}$ & $\begin{array}{l}9.53 \pm 3.2 \\
47.1 \pm 8.7\end{array}$ & $\begin{array}{c}9.68 \pm 5.3 \\
52.71 \pm 15.8\end{array}$ & $\begin{array}{l}t_{23.4}=-0.10 \\
t_{21.9}=-1.28\end{array}$ & $\begin{array}{l}p=0.93 \\
p=0.22\end{array}$ & $t_{20}=-18.4$ & $p<0.001$ & $t_{15}=-11.19$ & $p<0.001$ \\
\hline
\end{tabular}

Abbreviations: AMPT, alpha-methyl-paratyrosine; HC, healthy control participants; hit rate, proportion of successful key presses; rBN, remitted bulimic participants; reaction time, mean reaction time in successful trials.

Behavioral performance in the MID task in response to catecholamine depletion and sham depletion. Mean, standard deviation, and the results of two-tailed Student's or Welch's t-test are presented.

interaction (reaction time: $\mathrm{F}_{1,36}=0.53, \quad p=0.48 ;$ hit rate: $\left.\mathrm{F}_{1,36}=0.01, p=0.95\right)$. Analyzing group differences in reaction time and hit rate in response to different valences and magnitudes, we found a trend toward significance for the group-by-magnitude interaction $\left(\mathrm{F}_{1,38.5}=3.29, p=0.078\right)$ for the reaction time. Post hoc $t$-tests revealed that, over both drug conditions and both valences, HC participants showed a clear reduced reaction time after large relative to small monetary outcome anticipation $\left(t_{20}=2.97, p=0.008\right)$. In contrast, we found no significant magnitude-dependent difference in reaction time in $\mathrm{rBN}$ participants $\left(t_{16}=0.54\right.$, $p=0.60$ ). Concerning hit rate, the two groups showed a significant three-way interaction with the drug conditions and magnitudes of the monetary outcome (group-by-drugby-magnitude interaction: $\mathrm{F}_{1,288}=7.09, p=0.009$ ). In post hoc $t$-tests, we found that AMPT induced a reduced hit rate after large $\left(t_{20}=2.43, p=0.025\right)$, but not after small monetary outcome anticipation $\left(t_{20}=0.33, p=0.75\right)$ relative to the sham condition in HC participants. RBN participants showed a reversed pattern: Following AMPT, they had a reduced hit rate after small $\left(t_{16}=3.07, p=0.008\right)$ and not after large outcome anticipation $\left(t_{16}=-0.97, p=0.35\right)$.

The measurement of the serum prolactin level was missing following AMPT in one rBN participant. AMPT reduced significantly the serum prolactin levels in the other participants $\left(F_{1,35}=389.3, p<0.0001\right)$, but the two groups did not show a significant difference $\left(\mathrm{F}_{1,35}=1.64, p=0.21\right)$, and did not interact with the drug conditions significantly $\left(\mathrm{F}_{1,35}=1.79, p=0.19\right)$.

\section{Functional MR Imaging}

To examine the impact of AMPT on the neural activation in the AVS, we calculated a flexible factorial analysis including the contrast images 'large versus small monetary gain anticipation' of each participant and drug condition. This analysis revealed a significant main effect for the drug conditions in the left AVS. In detail, the difference between 
anticipating large relative to small monetary gain, namely the reward anticipation-related activation, was decreased in this region following AMPT in comparison to the sham condition $\left(\left[\begin{array}{lll}x & y & z\end{array}\right]=\left[\begin{array}{lll}-12 & 12 & -8\end{array}\right]\right.$; peak F-value: $\mathrm{F}_{1,36}=16.4$, $p=0.047$; peak $t$-value: $T_{36}=4.05 ; p=0.024$, FWE-small volume corrected). No significant group-by-drug interaction was found in our ROI.

In a similar manner, we calculated a further flexible factorial analysis, including the contrast images 'large versus small monetary loss anticipation' of each participant and drug condition. In the AVS, we did not find a significant main effect of drug condition and group-by-drug interaction.

Activation differences between the anticipation of large monetary gain or loss vs no outcome, and between anticipation of large $v s$ small monetary gain or loss in the sham condition are presented in the Supplementary Table S1a-d.

\section{Correlation between AMPT-Induced Changes of the Reward Anticipation-Related Neural Activation and Monetary Earning Alterations}

Moreover, we examined the relationship between AMPTdependent changes of the neural activation in the AVS and the monetary earning in the MID task. In HC participants, the AMPT-induced reward anticipation-related neural activation changes in the $4 \mathrm{~mm}$-sphere in the left AVS were significantly associated with the AMPT-induced changes in monetary earning $(r=0.54, p=0.012$; Figure $3 \mathrm{a})$. In detail, the more AMPT-reduced monetary earning relative to the sham condition, the higher was the AMPT-induced neural activation decrease between the reward magnitudes in the left AVS. RBN participants showed a trend toward a significantly negative association between AMPT-induced changes in the monetary earning and neural activation in the left AVS ( $r=-0.43, p=0.086$; Figure $3 b)$. Furthermore, the correlation coefficients were significantly different between both groups ( $z=2.99, p=0.003$, two-tailed).

\section{Associations between Characteristics of the Participants or Behavioral Ratings and AMPT-Induced Changes of the Reward Anticipation-Related Neural Activation or Monetary Earning}

Lastly, we assessed whether BMI, age, or depressive and eating disorder symptoms at the screening visit or during the most severe bulimic period in the past active illness of the rBN participants had an important influence on the impact of AMPT on neural activation of the AVS or monetary earning. These correlation analyses revealed no significant associations in rBN participants and we found no significant correlations between AMPT-dependent neural activation or monetary earning changes and BMI, age, or depressive symptoms in HC participants. Detailed results are provided in the Supplementary Information. As BMI was found to be significantly reduced in $\mathrm{rBN}$ relative to $\mathrm{HC}$ participants (Table 1), we analyzed a possible influence of BMI on the findings. These analyses revealed no significant influence of BMI. A detailed description is provided in the Supplementary Information.

The AMPT-induced changes of arousal or alertness, that is, the subscales vigor and fatigue of the POMS were not significantly associated with changes of monetary earning or reward anticipation-related neural activation in the AVS.

\section{Follow-Up Assessment}

Six $\mathrm{rBN}$ participants reported at least one binge eating or purging episode after study participation. In comparison, nine remitted $\mathrm{rBN}$ participants stayed in remission and two participants denied their participation in the follow-up telephone interview. RBN participants with and without relapse showed no differences regarding the impact of
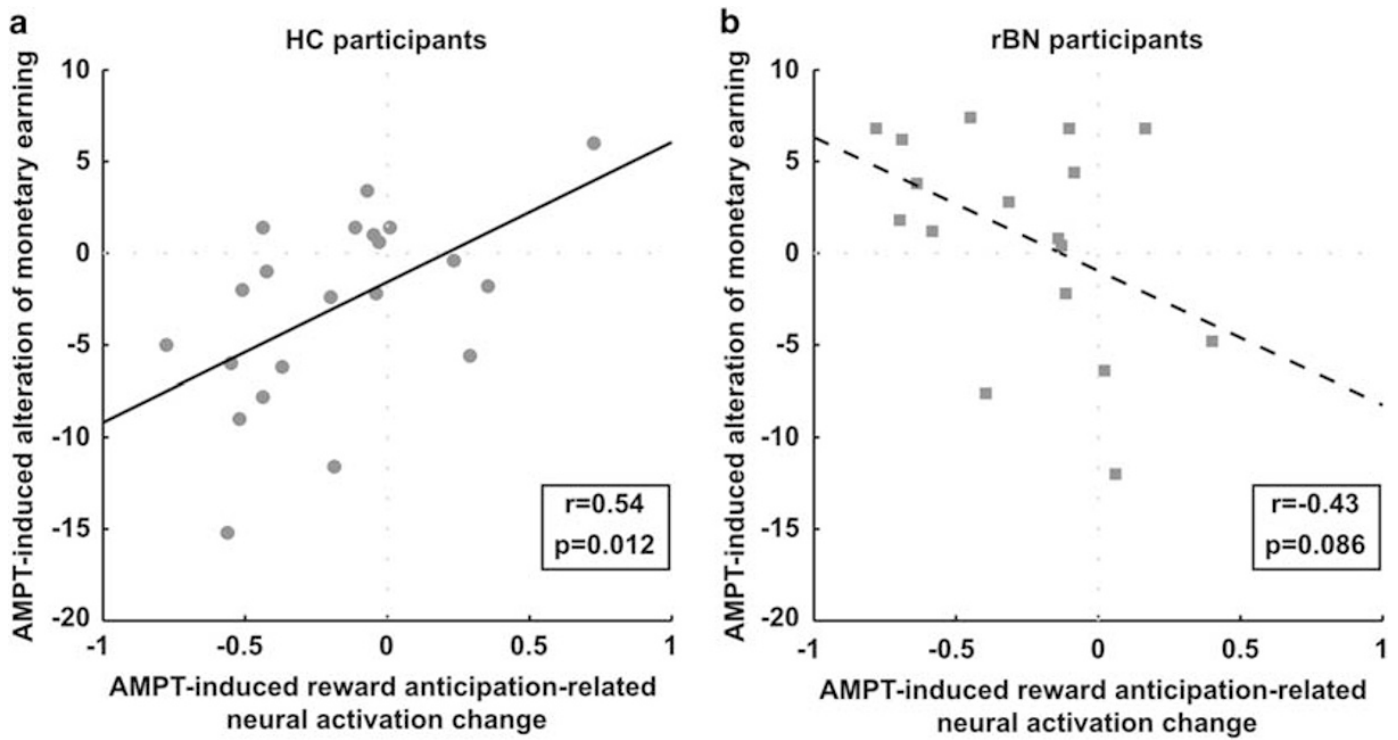

Figure 3 Scatter plot of the AMPT-induced alteration of the monetary earning and the AMPT-induced reward anticipation-related neural activation change in a sphere with a radius of $4 \mathrm{~mm}$ centered on the peak voxel in the left anteroventral striatum (AVS, MNI-coordinate $\left[\begin{array}{lll}x & y & z\end{array}\right]=\left[\begin{array}{llll}-12 & 12 & -8\end{array}\right]$ ) separately for (a) $\mathrm{HC}$ and (b) rBN participants. 
AMPT on their behavioral performance and reward anticipation-related neural activation in the left AVS. Detailed results are presented in the Supplementary Information.

\section{DISCUSSION}

This present study showed that catecholamine depletion had an impact on the reward-effort processing in healthy individuals: AMPT reduced the ability to integrate effectively reward magnitudes and effort costs to guide behavioral responses, which was indicated by decreased monetary earning. In remitted $\mathrm{BN}$, however, the reward-effort integration was not affected by catecholamine depletion. Importantly, rBN participants already showed a reduced effectiveness of reward-effort processing in the sham condition. Their behavioral performance in this drug condition was related to illness duration: A longer total duration of active illness was associated with a reduced monetary earning. Concerning the impact of catecholamine depletion on neural activation, AMPT reduced reward anticipation-related neural activation in the AVS in both groups. In healthy individuals, this AMPT-induced decrease of reward anticipation-related neural activation was associated with the AMPT-induced reduction of monetary earning. This relationship was found to be reversed in remitted BN. These findings indicate that catecholamines importantly influence behavioral and neural reward-effort processing in healthy individuals, whereas this association seems to be disrupted in remitted BN.

Regarding the effect of catecholamine depletion on behavioral performance in the MID task, previous studies revealed only an AMPT-induced reaction time increase in schizophrenic patients or participants remitted from MDD and not in healthy individuals (da Silva Alves et al, 2013, 2011; Hasler et al, 2009). In our study, however, both HC and rBN participants showed an increased reaction time following AMPT. A possible explanation for this discrepancy may be the continuous adaption of the target square duration according to the performance of the participants in our study. This adaption had probably an important impact on the reaction time. Further, healthy individuals and participants remitted from MDD showed no AMPT-induced alteration of the monetary earning contrary to our study (Hasler et al, 2009). This previous study, however, included higher incentive values and more trials, which led to a higher average and variance of the monetary earnings relative to our study. These distinctions may explain the different findings.

In general, higher monetary earnings result from an effective integration of reward magnitudes and effort costs during behavioral guidance. The reward-effort integration has been reported to be importantly related to the dopamine system: Dopamine depletion reduced the number of lever presses to obtain food relative to the effort costs in rats (Niv et al, 2007). Patients with Parkinson's disease showed less effort in an unmedicated relative to a medicated condition (Le Bouc et al, 2016). Our findings in healthy individuals are in line with these studies: AMPT induced a reduction of monetary earning. Hence, in healthy individuals, catecholamine depletion may have reduced the ability to take reward and effort into account to perform effectively.
Regarding the effect of catecholamine depletion on reward processing in remitted $\mathrm{BN}$, our previous study revealed that AMPT-induced reward learning deficits in $\mathrm{rBN}$ participants: AMPT reduced the propensity to make an effort to answer correctly often rewarded trials (Grob et al, 2012). In contrast, in our present study, which was based on a different participant sample, the reward-effort integration indicated by the monetary earning was found to be already reduced in rBN participants in the sham condition. This discrepancy in the findings might arise from the different tasks used in both studies. The discrimination task applied in our previous study might have been less dopamine-dependent than the MID task used in our present study. Therefore, differences between both groups in the discrimination task might be visible only after a reduction of catecholamine availability. In contrast, the strongly dopamine-dependent MID task might reveal a preexisting-reduced reward-effort integration in remitted $\mathrm{BN}$, probably leading to behavioral differences already in the sham condition. Importantly, a longer illness duration was related to a reduced monetary earning in the sham condition. This finding suggests that with a longer maintenance of bulimic symptoms, the effective reward-effort integration decreased more perhaps by further desensitizing of the dopaminergic system in BN (Frank, 2016).

The analysis of the $\mathrm{AMRI}$ data revealed that AMPT reduced the reward anticipation-related neural activation in the AVS in both groups. An earlier study revealed that the ventral striatum reflected the integration of reward magnitudes and effort costs in its activation (Croxson et al, 2009). A positron emission tomography study showed that dopamine release in the caudate, putamen, and ventral striatum was associated with increased monetary earning in a probabilistic reinforcement learning task (Kasanova et al, 2017). These results and the finding of dopamine depletion leading to a reduced propensity of effort-related behavior (Niv et al, 2007) suggest that catecholamines availability in the AVS might importantly affect the neural activation in response to different gain magnitudes. This suggestion fits to our finding that catecholamine depletion reduced the reward anticipationrelated neural activation of the AVS. In previous pharmacological challenge studies, however, an increased dopamine level induced by a single dose of dextroamphetamine or methylphenidate, respectively, reduced the neural activation in the ventral striatum during the anticipation of monetary gain relative to the placebo condition (Knutson et al, 2004; Schouw et al, 2013). In contrast, a prolonged administration of a combined serotonin-norepinephrine reuptake inhibitor induced an increased activation in the ventral striatum during the anticipation of monetary gain (Ossewaarde et al, 2011). Trying to distinguish the effect of enhanced levels of serotonin and norepinephrine, healthy individuals showed reduced neural activation in the ventral striatum in response to chocolate after a prolonged administration of a selective serotonin reuptake inhibitor relative to a selective norepinephrine reuptake inhibitor or placebo (McCabe et al, 2010). In addition, the intake of a phenylalanine/tyrosine-depleted amino-acid drink reducing catecholamines availability decreased activation in the striatum during a food rating task in healthy women (Frank et al, 2016). Taken together, most studies are consistent with our findings that catecholamine depletion leads to a weakening of the association between reward magnitude and neutral activation of the AVS. 
Studies reporting neural activation changes in the ventral striatum in response to different drug conditions described alterations in the contrast between the anticipation of monetary gain $v s$ no outcome (Knutson et al, 2004; Schouw et al, 2013). In this present study, however, we did not find a significant main effect of the drug conditions or a group-by-drug interaction in the AVS in this comparison. Nonetheless, our present study showed that catecholamine depletion reduced the reward anticipation-related neural activation in this region. This finding may indicate that the neural activation in the AVS is dependent on catecholamine availability in trials requiring the consideration of reward magnitudes to guide behavioral performance.

In line with the reports of the ventral striatum activation integrating reward magnitudes and effort costs (Croxson et al, 2009), and of the involvement of dopamine in effortrelated behavior (Niv et al, 2007), our study revealed that the reduced reward anticipation-related neural activation of the AVS following catecholamine depletion was significantly associated with the AMPT-induced decrease of monetary earning in healthy individuals. This means that the less the activation in the AVS differentiated between anticipating large and small monetary gains following AMPT, the higher was the AMPT-induced reduction of monetary earning. Accordingly, an earlier study revealed that the activation of the nucleus accumbens predicted in each trial the effort healthy individuals made in an instrumental motivation task (Kroemer et al, 2014). Therefore, we concluded that catecholaminergic neurotransmission is directly related to the reward-effort relationship influencing both neural activation of the AVS and effective behavioral performance in healthy individuals. RBN participants, however, showed no significant association between the activation in this region and the monetary earning. This finding is in line with Frank's theory on a desensitized dopaminergic system in BN (Frank, 2016). Consistently, a previous study showed that the activation of the AVS did not differentiate between positive and negative feedback in remitted BN (Wagner et al, 2010). Our findings revealed that the reward-effort integration to guide behavior effectively is probably disordered in remitted $\mathrm{BN}$ due to the desensitization of the dopaminergic system.

Some limitations of our study merit comment. First, AMPT reduces not specifically the dopamine availability. AMPT is a tyrosine hydroxylase inhibitor and consequently, reduces the synthesis of catecholamines which includes, beside dopamine, norepinephrine and epinephrine. As a result, we were not able to disentangle the responses to the depletion of these different catecholamines. Second, this study included an insufficient number of participants to detect differences between $\mathrm{rBN}$ and $\mathrm{HC}$ participants concerning the impact of valence and magnitude of the monetary outcome on reaction time and hit rate. Including more participants would increase the statistical power to be able to draw conclusion of interaction effects. Third, we were not able to test the impact of different phases of a normal menstrual cycle on our findings, because only nine participants included in this study were not using hormonal contraceptives. Moreover, the duration between the two experimental sessions differed largely between the participants and hence, might have influenced our findings. Nonetheless, the duration between the sessions was not significantly different between $\mathrm{HC}$ and $\mathrm{rBN}$ participants
(Supplementary Information). Therefore, we assumed that the different durations, if at all, had only a minor impact on our findings. Finally, the performance in the MID task may differentiate between the first and second session and therefore, the session order might have affected our findings. The analyses of the impact of the session order on rewardrelated behavior and reward anticipation-related neural activation, however, revealed no significant differences between the sessions (Supplementary Information). Hence, we assumed that no learning effect influenced our findings.

This study suggests that catecholaminergic neurotransmission is importantly related to the integration of reward magnitudes and effort costs to guide behavior effectively in healthy individuals. In remitted $\mathrm{BN}$, a reduced reward-effort integration was found to be related to illness duration but unrelated to catecholamine depletion. This finding is in line with Frank's theory of a desensitized dopaminergic system in BN (Frank, 2016). In addition, the AMPT-induced decrease of the reward anticipation-related neural activation in the AVS was unrelated to the reward-effort processing in remitted $\mathrm{BN}$ contrary to healthy individuals. A reduced effectiveness of integrating reward magnitudes and effort costs may lead to disadvantages and stress in daily life, which was reported to increase the probability of binge eating and purging behavior (Hardaway et al, 2015). Increasing the effectiveness of the reward-effort integration might be an appropriate method to increase the motivation for therapeutic treatment of $\mathrm{BN}$ (Hasler et al, 2004). Therefore, our findings encourage prospective studies on reward-effort processing in $\mathrm{BN}$.

\section{FUNDING AND DISCLOSURE}

This research was supported by the Swiss National Science Foundation (Nr. 32003B_138264). The authors declare no conflict of interest.

\section{ACKNOWLEDGMENTS}

We thank Dr Exadaktylos from the Department of Emergency Medicine, Dr Leichtle from the University Institute of Clinical Chemistry, and their teams at the University Hospital Inselspital, University of Bern, for blood sampling and measuring the serum prolactin levels.

\section{REFERENCES}

Balodis IM, Kober H, Worhunsky PD, White MA, Stevens MC, Pearlson GD et al (2013). Monetary reward processing in obese individuals with and without binge eating disorder. Biol Psychiatry 73: 877-886.

Bates D, Maechler M, Bolker B, Walker S (2015). Fitting linear mixed-effects models using lme4. J Stat Softw 67: 1-48.

Bohon C, Stice E (2011). Reward abnormalities among women with full and subthreshold bulimia nervosa: a functional magnetic resonance imaging study. Int J Eating Disord 44: 585-595.

Bremner JD, Vythilingam M, Ng CK, Vermetten E, Nazeer A, Oren DA et al (2003). Regional brain metabolic correlates of $\alpha$-methylparatyrosine-induced depressive symptoms: Implications for the neural circuitry of depression. JAMA 289: 3125-3134.

Croxson PL, Walton ME, O'Reilly JX, Behrens TEJ, Rushworth MFS (2009). Effort-based cost-benefit valuation and the human brain. J Neurosci 29: 4531-4541. 
da Silva Alves F, Bakker G, Schmitz N, Abeling N, Hasler G, van der Meer J et al (2013). Dopaminergic modulation of the reward system in schizophrenia: A placebo-controlled dopamine depletion fMRI study. Eur Neuropsychopharmacol 23: 1577-1586.

da Silva Alves F, Schmitz N, Figee M, Abeling N, Hasler G, van der Meer J et al (2011). Dopaminergic modulation of the human reward system: a placebo-controlled dopamine depletion fMRI study. J Psychopharmacol 25: 538-549.

DelDonno SR, Weldon AL, Crane NA, Passarotti AM, Pruitt PJ, Gabriel LB et al (2015). Affective personality predictors of disrupted reward learning and pursuit in major depressive disorder. Psychiatry Res 230: 56-64.

Drevets WC, Gautier C, Price JC, Kupfer DJ, Kinahan PE, Grace AA et al (2001). Amphetamine-induced dopamine release in human ventral striatum correlates with euphoria. Biol Psychiatry 49: 81-96.

Fiorillo CD, Tobler PN, Schultz W (2003). Discrete coding of reward probability and uncertainty by dopamine neurons. Science 299: 1898-1902.

First MB, Spitzer RL, Gibbon M, Williams JBW (2002). Structured Clinical Interview for DSM-IV-TR axis I disorders, research version, patient edition (SCID-I/P) Biometrics Research. New York State Psychiatric Institute: New York.

Frank GKW (2013). Altered brain reward circuits in eating disorders: chicken or egg? Curr Psychiatry Rep 15: 1-7.

Frank GKW (2016). The perfect storm - a biopsychosocial risk model for developing and maintaining eating disorders. Front Behav Neurosci 10: 44.

Frank GKW, Reynolds JR, Shott ME, O'Reilly RC (2011). Altered temporal difference learning in bulimia nervosa. Biol Psychiatry 70: 728-735.

Frank S, Veit R, Sauer H, Enck P, Friederich H-C, Unholzer T et al (2016). Dopamine depletion reduces food-related reward activity independent of BMI. Neuropsychopharmacology 41: 1551-1559.

Grob S, Pizzagalli DA, Dutra SJ, Stern J, Morgeli H, Milos G et al (2012). Dopamine-related deficit in reward learning after catecholamine depletion in unmedicated, remitted subjects with bulimia nervosa. Neuropsychopharmacology 37: 1945-1952.

Hardaway JA, Crowley NA, Bulik CM, Kash TL (2015). Integrated circuits and molecular components for stress and feeding: implications for eating disorders. Genes Brain Behav 14: 85-97.

Harrell FEJ (2016). Hmisc: Harrell miscellaneous, $R$ package version, 40-42.

Hasler G (2012). Can the neuroeconomics revolution revolutionize psychiatry? Neurosci Biobehav Re 36: 64-78.

Hasler G, Delsignore A, Milos G, Buddeberg C, Schnyder U (2004). Application of Prochaska's transtheoretical model of change to patients with eating disorders. J Psychosom Res 57: 67-72.

Hasler G, Luckenbaugh DA, Snow J, Meyers N, Waldeck T, Geraci $M$ et al (2009). Reward processing after catecholamine depletion in unmedicated, remitted subjects with major depressive disorder. Biol Psychiatry 66: 201-205.

Kasanova Z, Ceccarini J, Frank MJ, Amelsvoort Tv, Booij J, Heinzel A et al (2017). Striatal dopaminergic modulation of reinforcement learning predicts reward-oriented behavior in daily life. Biol Psychol 127: 1-9.

Knutson B, Adams CM, Fong GW, Hommer D (2001a). Anticipation of increasing monetary reward selectively recruits nucleus accumbens. J Neurosci 21: RC 159.

Knutson B, Bjork JM, Fong GW, Hommer D, Mattay VS, Weinberger DR (2004). Amphetamine modulates human incentive processing. Neuron 43: 261-269.

Knutson B, Fong GW, Adams CM, Varner JL, Hommer D (2001b). Dissociation of reward anticipation and outcome with eventrelated fMRI. NeuroReport 12: 3683-3687.
Knutson B, Heinz A (2015). Probing psychiatric symptoms with the monetary incentive delay task. Biol Psychiatry 77: 418-420.

Kroemer NB, Guevara A, Ciocanea Teodorescu I, Wuttig F, Kobiella A, Smolka MN (2014). Balancing reward and work: anticipatory brain activation in NAcc and VTA predict effort differentially. NeuroImage 102: 510-519.

Kuznetsova A, Brockhoff PB, Christensen RHB (2016). lmerTest: tests in linear mixed effects models. $R$ package version $20-33$.

Lam RW, Tam EM, Grewal A, Yatham LN (2001). Effects of alphamethyl-para-tyrosine-induced catecholamine depletion in patients with seasonal affective disorder in summer remission. Neuropsychopharmacology 25(5, Supplement 1): S97-S101.

Le Bouc R, Rigoux L, Schmidt L, Degos B, Welter M-L, Vidailhet M et al (2016). Computational dissection of dopamine motor and motivational functions in humans. J Neurosci 36: 6623-6633.

McCabe C, Mishor Z, Cowen PJ, Harmer CJ (2010). Diminished neural processing of aversive and rewarding stimuli during selective serotonin reuptake inhibitor treatment. Biol Psychiatry 67: 439-445.

McNair DM, Lorr M, Droppleman LF (1992). Profile of Mood States: POMS EdiTS, Educational and Industrial Testing Service. Calif: San Diego.

Mueller SV, Mihov Y, Federspiel A, Wiest R, Hasler G (2017). Neural response to catecholamine depletion in remitted bulimia nervosa: Relation to depression and relapse. Eur Neuropsychopharmacol 27: 633-646.

Neumeister A, Turner EH, Matthews JR, Postolache TT, Barnett RL, Rauh $\mathrm{M}$ et al (1998). Effects of tryptophan depletion vs catecholamine depletion in patients with seasonal affective disorder in remission with light therapy. Arch Gen Psychiatry 55: 524-530.

Niv Y, Daw ND, Joel D, Dayan P (2007). Tonic dopamine: opportunity costs and the control of response vigor. Psychopharmacology 191: 507-520.

Ossewaarde L, Verkes RJ, Hermans EJ, Kooijman SC, Urner M, Tendolkar I et al (2011). Two-week administration of the combined serotonin-noradrenaline reuptake inhibitor duloxetine augments functioning of mesolimbic incentive processing circuits. Biol Psychiatry 70: 568-574.

Psychology Software Tools (Pittsburgh, PA). Inc. [E-prime 2.0] (2012). Retrieved from http://pstnet.com.

R Foundation for Statistical Computing (2017) R: A Language And Environment For Statistical Computing. R Foundation for Statistical Computing: Vienna, Austria.

Salamone JD, Correa M, Farrar A, Mingote SM (2007). Effortrelated functions of nucleus accumbens dopamine and associated forebrain circuits. Psychopharmacology 191: 461-482.

Schmidtke A, Fleckenstein P, Moises W, Beckmann H (1988). Studies of the reliability and validity of the German version of the Montgomery-Asberg Depression Rating Scale (MADRS). Schweizer Archiv fur Neurologie und Psychiatrie (Zurich, Switzerland: 1985) 139: 51-65.

Schouw MLJ, Kaag AM, Caan MWA, Heijtel DFR, CBLM Majoie, Nederveen AJ et al (2013). Mapping the hemodynamic response in human subjects to a dopaminergic challenge with dextroamphetamine using ASL-based pharmacological MRI. Neurolmage 72: 1-9.

Schultz W (2016). Reward functions of the basal ganglia. J Neural Transm 123: 679-693.

Wagner A, Aizenstein H, Venkatraman VK, Bischoff-Grethe A, Fudge J, May JC et al (2010). Altered striatal response to reward in bulimia nervosa after recovery. Int J Eating Disord 43: 289-294.

Wierenga CE, Ely A, Bischoff-Grethe A, Bailer UF, Simmons AN, Kaye WH (2014). Are extremes of consumption in eating disorders related to an altered balance between reward and inhibition? Front Behav Neurosci 8: 410.

Supplementary Information accompanies the paper on the Neuropsychopharmacology website (http://www.nature.com/npp) 